\title{
Measurements of Deuteron Photodisintegration up to 4.0 GeV
}

C. Bochna, ${ }^{1}$ B. P. Terburg, ${ }^{1}$ D. J. Abbott,${ }^{2}$ A. Ahmidouch,${ }^{3}$ C. S. Armstrong, ${ }^{4}$ J. Arrington, ${ }^{5}$ K. A. Assamagan, ${ }^{6}$ O. K. Baker, ${ }^{2,6}$ S. P. Barrow, ${ }^{7}$ D. P. Beatty,${ }^{7}$ D. H. Beck, ${ }^{1}$ S. Y. Beedoe,${ }^{8}$ E. J. Beise, ${ }^{9}$ J.E. Belz, ${ }^{10}$ P. E. Bosted,${ }^{11}$ E. J. Brash, ${ }^{12,18}$ H. Breuer, ${ }^{9}$ R. V. Cadman, ${ }^{1}$ L. Cardman, ${ }^{2}$ R. D. Carlini, ${ }^{2}$ J. Cha, ${ }^{6}$ N. S. Chant, ${ }^{9}$ G. Collins, ${ }^{9}$ C. Cothran, ${ }^{13}$ W. J. Cummings, ${ }^{14}$ S. Danagoulian, ${ }^{8}$ F. A. Duncan, ${ }^{9}$ J. A. Dunne, ${ }^{2}$ D. Dutta, ${ }^{15}$ T. Eden,${ }^{6}$ R. Ent, ${ }^{2}$ B. W. Filippone, ${ }^{5}$ T. A. Forest, ${ }^{1}$ H. T. Fortune, ${ }^{7}$ V. V. Frolov, ${ }^{16}$ H. Gao, ${ }^{1,17}$ D. F. Geesaman, ${ }^{14}$ R. Gilman, ${ }^{18}$ P. L. J. Gueye, ${ }^{6}$ K. K. Gustafsson, ${ }^{9}$ J.-O. Hansen, ${ }^{14}$ M. Harvey, ${ }^{6}$ W. Hinton, ${ }^{6}$ R. J. Holt, ${ }_{1}^{1}$ H. E. Jackson, ${ }^{14}$ C. E. Keppel, ${ }^{6}$ M. A. Khandaker, ${ }^{19}$ E. R. Kinney, ${ }^{20}$ A. Klein, ${ }^{21}$ D. M. Koltenuk, ${ }^{7}$ G. Kumbartzki, ${ }^{17}$ A. F. Lung, ${ }^{9}$ D. J. Mack, ${ }^{2}$ R. Madey ${ }^{3,6}$ P. Markowitz, ${ }^{22}$ K. W. McFarlane, ${ }^{19}$ R. D. McKeown, ${ }^{5}$ D. G. Meekins, ${ }^{4}$ Z.-E. Meziani, ${ }^{23}$ M. A. Miller, ${ }^{1}$ J. H. Mitchell, ${ }^{2}$ H. G. Mkrtchyan, ${ }^{24}$ R. M. Mohring, ${ }^{9}$ J. Napolitano, ${ }^{16}$ A. M. Nathan, ${ }^{1}$ G. Niculescu, ${ }^{6}$ I. Niculescu, ${ }^{6}$ T. G. O'Neill, ${ }^{14}$ B. R. Owen, ${ }^{1}$ S. F. Pate, ${ }^{25}$ D. H. Potterveld,${ }^{14}$ J. W. Price, ${ }^{16}$ G. L. Rakness, ${ }^{20}$ R. Ransome,${ }^{18}$ J. Reinhold, ${ }^{14}$ P. M. Rutt, ${ }^{18}$ C. W. Salgado, ${ }^{19}$ G. Savage,${ }^{6}$ R. E. Segel,${ }^{15}$ N. Simicevic,${ }^{1}$ P. Stoler, ${ }^{16}$ R. Suleiman, ${ }^{3}$ L. Tang, ${ }^{6}$ D. van Westrum, ${ }^{20}$ W. F. Vulcan, ${ }^{2}$ S. Williamson, ${ }^{1}$ M. T. Witkowski, ${ }^{16}$ S. A. Wood, ${ }^{2}$ C. Yan, ${ }^{2}$ and B. Zeidman ${ }^{14}$

${ }^{1}$ University of Illinois at Urbana-Champaign, Urbana, Illinois 61801

${ }^{2}$ Thomas Jefferson National Accelerator Facility, Newport News, Virginia 23606

${ }^{3}$ Kent State University, Kent, Ohio 44242

${ }^{4}$ College of William and Mary, Williamsburg, Virginia 23187

${ }^{5}$ California Institute of Technology, Pasadena, California 91125 ${ }^{6}$ Hampton University, Hampton, Virginia 23668

${ }^{7}$ University of Pennsylvania, Philadelphia, Pennsylvania 19104

${ }^{8}$ North Carolina A\&T State University, Greensboro, North Carolina 27411

${ }^{9}$ University of Maryland, College Park, Maryland 20742

${ }^{10}$ TRIUMF, Vancouver, British Columbia, Canada V6T 2A3

${ }^{11}$ American University, Washington, D.C. 20016

${ }^{12}$ University of Regina, Regina, Saskatchewan, Canada S4S OA2

${ }^{13}$ University of Virginia, Charlottesville, Virginia 22901

${ }^{14}$ Argonne National Laboratory, Argonne, Illinois 60439

${ }^{15}$ Northwestern University, Evanston, Illinois 60201

${ }^{16}$ Rensselaer Polytechnic Institute, Troy, New York 12180

${ }^{17}$ Massachusetts Institute of Technology, Cambridge, Massachusetts 02139

${ }^{18}$ Rutgers University, New Brunswick, New Jersey 08903

${ }^{19}$ Norfolk State University, Norfolk, Virginia 23504

${ }^{20}$ University of Colorado, Boulder, Colorado 80309

${ }^{21}$ Old Dominion University, Norfolk, Virginia 23529

${ }^{22}$ Florida International University, University Park, Florida 33199

${ }^{23}$ Temple University, Philadelphia, Pennsylvania 19122

${ }^{24}$ Yerevan Physics Institute, Yerevan, Armenia

${ }^{25}$ New Mexico State University, Las Cruces, New Mexico 88003

(Received 7 August 1998)

The first measurements of the differential cross section for the $d(\gamma, p) n$ reaction up to $4.0 \mathrm{GeV}$ were performed at the Continuous Electron Beam Accelerator Facility (CEBAF) at Thomas Jefferson Laboratory. We report the cross sections at the proton center-of-mass angles of $36^{\circ}, 52^{\circ}, 69^{\circ}$, and $89^{\circ}$. These results are in reasonable agreement with previous measurements at lower energy. The $89^{\circ}$ and $69^{\circ}$ data show constituent-counting-rule behavior up to $4.0 \mathrm{GeV}$ photon energy. The $52^{\circ}$ and $36^{\circ}$ data disagree with the counting-rule behavior. The quantum chromodynamics (QCD) model of nuclear reactions involving reduced amplitudes disagrees with the present data. [S0031-9007(98)07676-5]

PACS numbers: 25.20. $-\mathrm{x}, 13.75 . \mathrm{Cs}, 24.85 .+\mathrm{p}, 25.10 .+\mathrm{s}$

To reconcile low energy and high energy descriptions of hadronic matter, nuclear physics must determine when it is justified to make a transition from meson-nucleon degrees of freedom to quark-gluon degrees of freedom in the description of a nuclear reaction. The QCD content of nuclei was studied first by Brodsky and Chertok [1]. A possible signature for this transition is that the reaction cross section begins to scale at some incident energy. If scaling were indeed observed, characterization of the approach to scaling would be essential to understand how the dynamics are simplified. High energy twobody photodisintegration of the deuteron $(\gamma d \rightarrow p n)$ is 
particularly well suited for these studies because it is amenable to theoretical calculation, and relatively high momentum transfer to the constituents can be achieved at relatively modest photon energies [2].

Previous measurements [3,4] for the $d(\gamma, p) n$ reaction indicate the onset of scaling behavior at a photon energy of $1 \mathrm{GeV}$ at a reaction angle of $\theta_{\text {c.m. }}=90^{\circ}$; however, this limited data set does not show scaling at other reaction angles. Measurement of the angular distributions of scaling thresholds is an important part of characterizing the reaction process. In this Letter, we present new data to address this issue.

Predictions for the energy dependence of the cross section at high energies are given by constituent counting rules [5], the reduced nuclear amplitude analysis (RNA) [6], the quark-gluon string model (QGS) [7], and the asymptotic meson-exchange model (AMEC) [8]. Constituent counting rules predict that the energy dependence for the two-body exclusive reaction cross section should be given by

$$
\frac{d \sigma}{d t}=\frac{h\left(\theta_{\text {c.m. }}\right)}{s^{n-2}},
$$

where the Mandelstam variables $s$ and $t$ are the square of the total energy in the center-of-mass frame and the momentum transfer squared in the $s$ channel, respectively. The symbol $n$ denotes the total number of elementary fields in the initial and final states, and $n-2$ is 11 for the $d(\gamma, p) n$ reaction. The quantity $h\left(\theta_{\text {c.m. }}\right)$ depends on details of the dynamics of the process. Constituent counting rules [5] are believed valid at energies much greater than the masses of the participating particles, perhaps in the perturbative QCD region. The previous data at $90^{\circ}$ scale according to these rules with a photon energy threshold of only $1 \mathrm{GeV}$.

In the RNA approach, the amplitude is described in terms of parton exchange between the two nucleons. The low energy components responsible for quark binding within the nucleons are removed by dividing out the empirical nucleon form factors. While the reduced nuclear amplitude analysis appears to describe the electron-deuteron elastic scattering cross section above a momentum transfer of $1 \mathrm{GeV} / c$ [1], it does not give a good description of the previous deuteron photodisintegration data. This is surprising because this model is expected to approach scaling at lower energies than the constituent counting rules. The QGS model is based on Regge phenomenology and is expected to be valid at small values of $t$ where the parameters are best determined. Thus, the QGS model is expected to be valid at small reaction angles, while most of the existing data is at large angles.

The traditional meson-exchange models $[9,10]$ describe the data at energies below $1 \mathrm{GeV}$, but are problematic above $1 \mathrm{GeV}$. However, the AMEC [8] departs from the conventional approach in that an asymptotic description of the nucleon-nucleon interaction is used [11]. Although this model appears to predict the observed energy dependence at $\theta_{\text {c.m. }}=90^{\circ}$, it cannot yet reproduce the magnitude of the cross section.

In this Letter we present new results from the $d(\gamma, p) n$ reaction at proton center-of-mass angles of $36^{\circ}, 52^{\circ}$, $69^{\circ}$, and $89^{\circ}$. These data overlap with existing Stanford Linear Accelerator Center (SLAC) measurements, extending them to higher energy and providing more complete angular coverage.

Because the cross sections are much less than $1 \mathrm{nb} / \mathrm{sr}$, it was essential to have a high-current, high duty factor electron beam of multi-GeV energy, and to use wellshielded spectrometers of a large solid angle. This was achieved by performing the experiment of Hall $\mathrm{C}$ at Thomas Jefferson National Accelerator Facility (Jefferson $\mathrm{Lab}$ ). A $25 \mu \mathrm{A}$ continuous-wave electron beam in the energy range 0.845 to $4.045 \mathrm{GeV}$ in steps of $0.8 \mathrm{GeV}$ was incident on a $6 \%$ copper radiator to create an untagged photon beam. The resulting electron and bremsstrahlung beam impinged on a $15-\mathrm{cm}$ liquid deuterium target. Because the $\gamma d \rightarrow p n$ reaction is a two-body process, the photon energy could be reconstructed from the measured final-state proton momentum and scattering angle. Events with pion production were excluded by accepting only the protons with the highest momenta. Protons were detected with the High Momentum Spectrometer (HMS) [12].

The HMS had a solid angle of $6.8 \mathrm{msr}$ and a momentum acceptance of $18 \%$. The detector system consisted of a plastic scintillator hodoscope, drift chambers, and a gas Čerenkov counter. The hodoscope was used to form a trigger and also to provide the time-of-flight information for particle identification. Drift chambers were employed to measure particle trajectories in order to calculate the momenta and reaction angles. It is important to distinguish protons from pions and deuterons. A gas Čerenkov counter, filled with $\mathrm{C}_{4} \mathrm{~F}_{10}$ gas, was used in combination with time-of-flight (TOF) information to separate pions and protons above $2.8 \mathrm{GeV} / c$, while TOF alone was used for pion rejection at lower momenta. Deuteron rejection was accomplished by a TOF cut at all momenta.

Background contributions from the target windows were removed by placing cuts on the reconstructed target position and subtracting the yield obtained with a cell of identical dimensions that was either empty or filled with liquid hydrogen to simulate bremsstrahlung in the deuterium. This target length cut led to a $3 \%$ error for the target thickness. Deuterium and empty target data were taken alternately during the experiment. Data were taken with the hydrogen target at some of the kinematic settings to cross-check the procedure of the empty target subtraction. The yield from electrodisintegration was measured by repeating the procedure without the radiator present. This yield, approximately $20 \%$ of the total, was treated as a background and was subtracted from the photodisintegration yield with an energy-dependent correction factor to take into account the modification of the electron beam flux and energy distribution by the 
radiator [13]. An additional systematic error of 3\% was assigned to this background subtraction procedure.

The photon energy bin limits were chosen to kinematically eliminate photons from photopion production processes, and to eliminate the bremsstrahlung end point, for which the photon flux is less well known. This photon energy bin varied in width from $60 \mathrm{MeV}$ at $36^{\circ}$ to $115 \mathrm{MeV}$ at $89^{\circ}$. The bremsstrahlung photon flux was calculated with an estimated $3 \%$ uncertainty using the thick-target bremsstrahlung computer code developed by Belz [13], which was cross-checked by using the codes of Matthews and Owens [14]. The spectrometer solid angle for the extended target was studied by comparing a Monte Carlo simulation with measurements made with both a movable solid carbon target and the extended cryotargets. In addition, the dependence of the results on the choice of optics parameters for the HMS was studied. An overall error of $7 \%$ in the HMS acceptance was determined from these studies. A proton absorption correction was applied to compensate for the scattering in the spectrometer windows and the detector stack. This proton attenuation was measured [15] to be $(5.5 \pm 2.0) \%$ by comparing singles $\mathrm{H}\left(e, e^{\prime}\right)$ and coincidence $\mathrm{H}\left(e, e^{\prime} p\right)$ measurements. Corrections were also applied for the computer dead time and the tracking efficiency.

The overall systematic uncertainty is found to be $\leq 11.5 \%$. The uncertainties from the beam current measurement, beam energy determination, and photon energy reconstruction in the measured quantity $s^{11} d \sigma / d t$ were less than $3 \%$. The uncertainty from the particle identification is $55 \%$. An additional systematic error of $3 \%$ resulted from using a reduced solid angle in the HMS to avoid an obstruction from an HMS vacuum valve that was inadvertently partially closed $[16,17]$. Thus, only half of the HMS solid angle was used in the analysis of the data. A separate experiment without the obstruction verified that there was good agreement between the half acceptance of the experiment with the obstruction and the corresponding half acceptance for the experiment without the obstruction. A Monte Carlo simulation was used to determine the correction for "in-scattered" protons from the valve into the open half of the spectrometer solid angle. The amount of this correction is less than $2 \%$ in the worst case. This selection of half the spectometer solid angle resulted in a reduction of the observed reaction center-of-mass angle by approximately $1^{\circ}$ from the central spectrometer setting.

Figure 1 shows the world data of $s^{11} d \sigma / d t$ above $\sim 0.4 \mathrm{GeV}$ for the $\gamma d \rightarrow p n$ reaction at $\theta_{\text {c.m. }}=36^{\circ}, 52^{\circ}$, $69^{\circ}$, and $89^{\circ}$ as a function of the photon energy. The $89^{\circ}$ data are shown in the top panel of Fig. 1. The SLAC NE17 data [4] exhibit scaling behavior starting at photon energies around $1 \mathrm{GeV}$, corresponding to proton transverse momenta $\left(p_{T}\right)$ of $\sim 1.0 \mathrm{GeV} / c$. For the $d(\gamma, p) n$ reaction, $p_{T}^{2}$ can be expressed as $\frac{1}{2} E_{\gamma} M_{d} \sin ^{2}\left(\theta_{\text {c.m. }}\right)$, with $M_{d}$ being the deuteron mass. The Jefferson Lab data are in good agreement with the previous SLAC data. The

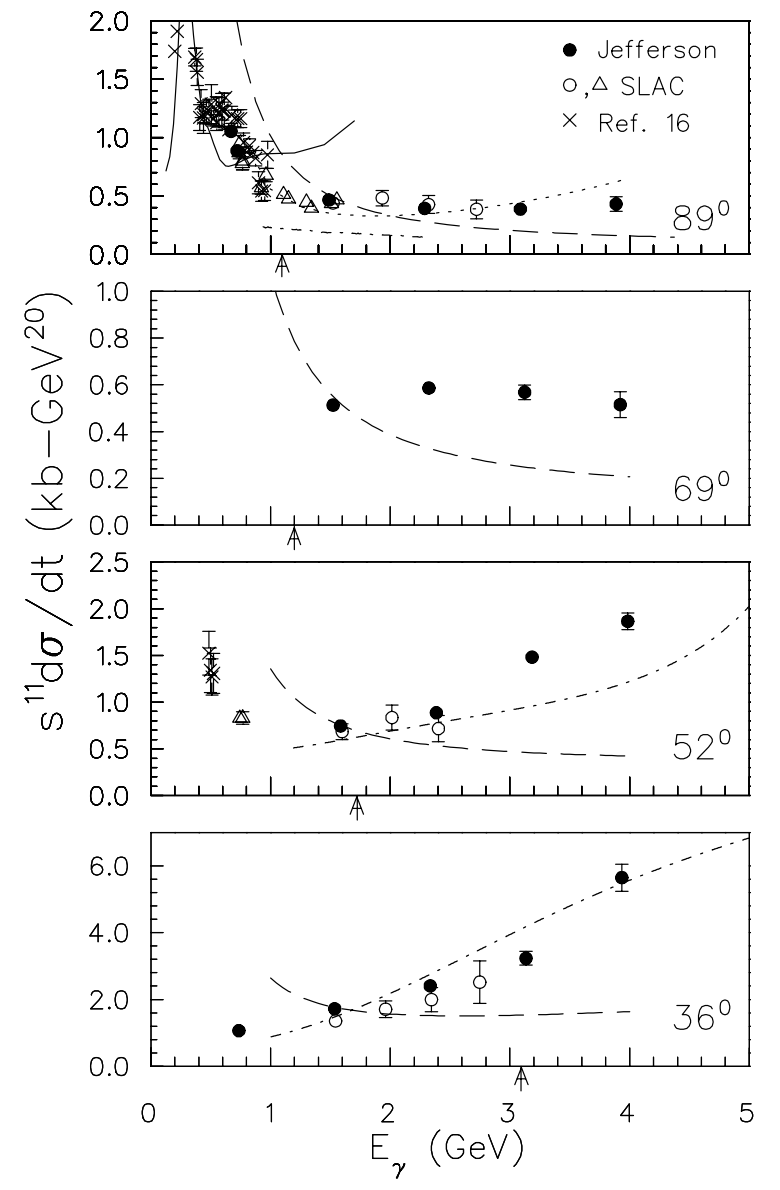

FIG. 1. $s^{11} d \sigma / d t$ vs $E_{\gamma}$. The present work is shown as solid circles with statistical uncertainties only, the SLAC NE17 data are shown as open circles, the SLAC NE8 data are shown as open triangles, and other existing data [18] are shown as crosses. The solid line is the meson-exchange model calculation of Lee [9]. The long-dashed line is the RNA analysis [6], and the dotted line is Nagornyi's [8] asymptotic meson-exchange calculation. The dash-dotted line is the QGS calculation [7]. The arrows indicate the photon energies where $p_{T}{ }^{2}=1.0(\mathrm{GeV} / c)^{2}$. The previous data are shown above at nominal center-of-mass angles of $37^{\circ}, 53^{\circ}$, and $90^{\circ}$.

new data continue to show the scaling behavior up to the highest photon energy of $4.0 \mathrm{GeV}$. The differential cross section $d \sigma / d t$ from the present work at photon energies above $1.0 \mathrm{GeV}$ were fitted with the form of $A / s^{n-2}$, and $n-2=11.1 \pm 0.3$ was obtained from the fit. It is surprising that the counting rule appears to work so well considering the fact that the momentum transfer to the individual quark is below $1.0(\mathrm{GeV} / c)^{2}$ for these measurements, where the strong coupling constant still varies significantly as a function of the momentum transfer.

The long-dashed line in Fig. 1 represents a reduced nuclear amplitude analysis [6] with a normalization factor chosen to agree with the data at $E_{\gamma}=1.6 \mathrm{GeV}$. This RNA curve falls below the high $E_{\gamma}$ data and does not reach an asymptotic limit at these energies. The QGS calculation [7] is shown as the dash-dotted line. The solid line represents the meson-exchange calculation of Lee [9], which is a standard calculation that reproduces 
the measured $N N$ phase shifts up to $2.0 \mathrm{GeV}$ and is also constrained by photomeson [19] production data. The calculation gives a reasonable description of the data below $0.5 \mathrm{GeV}$, but deviates above $1.0 \mathrm{GeV}$. The meson-exchange model calculation of Laget [10] (not shown) reproduces the experimental data fairly well from the threshold up to about $0.6 \mathrm{GeV}$. The dotted line is a calculation (AMEC) by Nagornyi et al. [8] with the normalization performed at $1.0 \mathrm{GeV}$. Although this calculation gives a different energy dependence to the cross section than that of the constituent counting rule, it does give an energy dependence very close to $1 / s^{11}$ in the energy region of 1.5 to $3.5 \mathrm{GeV}$. More calculations of this type are necessary at the other angles.

The data at $52^{\circ}$ and $69^{\circ}$ are shown in the center panels of Fig. 1. The $69^{\circ}$ data appear to scale according to the constituent counting rules. A fit to this cross section gives $n-2=10.9 \pm 0.2$ in good agreement with that at $89^{\circ}$. At $52^{\circ}$ the present data are in reasonable agreement with previous SLAC measurement and have significantly improved statistical errors. The results deviate from the scaling behavior predicted by counting rules. A fit of the scaling formula to the cross section data gives $n-2=$ $9.5 \pm 0.2$. The QGS calculation [7] is in fair agreement with the data in the photon energy region between 1.0 to 4.0 GeV.

The bottom panel in Fig. 1 shows the data at $\theta_{\text {c.m. }}=$ $36^{\circ}$. Fitting the scaling formula to the Jefferson Lab cross section data above a photon energy of $1 \mathrm{GeV}$ gives $n-2=9.6 \pm 0.1$. The highest $p_{T}$ from NE17 measurements at this $\theta_{\text {c.m. }}$ is $0.7 \mathrm{GeV} / c$. The experimental uncertainties and the limited energy region covered by the NE17 experiment preclude any conclusion with regard to the scaling behavior in the photon energy range below $3 \mathrm{GeV}$. The Jefferson Lab data are in reasonable agreement with the SLAC data [4] when the large angular dependence at forward angles is taken into account. The SLAC data were taken at $36.7^{\circ}$, while the present data were recorded from $35.4^{\circ}$ at the highest energy to $36.2^{\circ}$ at the lowest energy. The previous data shown in Fig. 1 are not corrected for this difference in angles.

Although a photon energy of $3.1 \mathrm{GeV}$ at $36^{\circ}$ corresponds to the same $p_{T}$ where the onset of $s^{-11}$ scaling is observed at $90^{\circ}$, there is no evidence from the $36^{\circ}$ data that scaling has set in (see arrows in Fig. 1). The RNA [6] deviates significantly from the data at $36^{\circ}$, but the QGS calculation [7] gives a reasonable energy dependence.

In summary, the data at $\theta_{\text {c.m. }}=89^{\circ}-90^{\circ}$ continue to show scaling behavior up to $4 \mathrm{GeV}$. The first evidence is observed for a similar scaling behavior at $69^{\circ}$. The asymptotic meson-exchange model is in fair agreement with the $90^{\circ}$ data in the photon energy region of 1.0 to $3.0 \mathrm{GeV}$. Thus far, no constituent counting scaling behavior is observed for the $36^{\circ}$ or $52^{\circ}$ data. The new data at $36^{\circ}$ and $52^{\circ}$ rule out a scaling threshold of $p_{T}=$ $1.0(\mathrm{GeV} / c)^{2}$. The reduced nuclear amplitude analysis does not agree with the present data. The QGS model has an energy dependence that is in fair agreement with the present data at $36^{\circ}$ and $52^{\circ}$. Further measurements at higher energies at the forward angles will be essential to test for scaling. Experimental determination of the physical observable associated with the onset of scaling is essential for understanding the underlying mechanism for the scaling behavior in the exclusive two-body process.

We acknowledge the outstanding work of the staff of the accelerator division at the Thomas Jefferson National Accelerator Facility in delivering the high quality electron beam to $4 \mathrm{GeV}$ and the Hall $\mathrm{C}$ technical staff for constructing the spectrometers and the cryotarget. We thank E. Schulte for bremsstrahlung calculations. This work is supported in part by the research grants from the U.S. Department of Energy and the U.S. National Science Foundation.

[1] S. J. Brodsky and B. T. Chertok, Phys. Rev. D 14, 3003 (1976); Phys. Rev. Lett. 37, 269 (1976).

[2] R. J. Holt, Phys. Rev. C 41, 2400 (1990).

[3] J. Napolitano et al., Phys. Rev. Lett. 61, 2530 (1988); S. J. Freedman et al., Phys. Rev. C 48, 1864 (1993).

[4] J. E. Belz et al., Phys. Rev. Lett. 74, 646 (1995).

[5] S. J. Brodsky and G. R. Farrar, Phys. Rev. Lett. 31, 1153 (1973); V. Matveev et al., Nuovo Cimento Lett. 7, 719 (1973); G. P. Lepage and S. J. Brodsky, Phys. Rev. D 22, 2157 (1980).

[6] S. J. Brodsky and J. R. Hiller, Phys. Rev. C 28, 475 (1983).

[7] L. A. Kondratyuk et al., Phys. Rev. C 48, 2491 (1993).

[8] S. I. Nagornyi, Yu. A. Kasatkin, and I. K. Kirchenko, Sov. J. Nucl. Phys. 55, 189 (1992); S. I. Nagornyi (private communication).

[9] T.-S.H. Lee, Argonne National Laboratory Report No. PHY-5253-TH-88; T.-S. H. Lee, in Procedings of the International Conference on Medium and High Energy Nuclear Physics, Taipei, Taiwan, 1988 (World Scientific, Singapore, 1988), p. 563.

[10] J. M. Laget, Nucl. Phys. A312, 265 (1978).

[11] W. W. Buck and F. Gross, Phys. Rev. D 20, 2361 (1979); F. Gross and B. D. Keister, Phys. Rev. C 28, 823 (1983).

[12] C. Yan et al., CEBAF Technical Note No. R-92-002 (1992).

[13] J. E. Belz, Ph.D. thesis, California Institute of Technology, 1994 (unpublished).

[14] J. L. Matthews and R. O. Owens, Nucl. Instrum. Methods 111, 157 (1973).

[15] D. van Westrum, Ph.D. thesis, University of Colorado, 1998 (unpublished).

[16] C. Bochna, Ph.D. thesis, University of Illinois (unpublished).

[17] B. P. Terburg, Ph.D. thesis, University of Illinois (unpublished).

[18] P. Dougan et al., Z. Phys. A 276, 55 (1976); R. Ching and C. Schaerf, Phys. Rev. 141, 1320 (1966); H. Myers et al., Phys. Rev. 121, 630 (1961); J. Arends et al., Nucl. Phys. A412, 509 (1984).

[19] T. Sato and T.-S. H. Lee, Phys. Rev. C 54, 2660 (1996). 